\title{
Estudio comparativo de los prospectos farmacéuticos franceses y españoles. Su aplicación en la formación de traductores biosanitarios
}

\author{
Concepción Mira Rueda (Málaga)
}

\begin{abstract}
The pharmaceutical industry is characterised by its success and strong stability in the international market even in times of crisis. That is to say that the pharmaceutical field could be an attractive source of employment for future translators and interpreters whose expertise, interest and knowledge are focused on medicine. This is the reason why it is extremely important to have a basic background of the medical field. In short, this paper pursues two goals: firstly, we study the main characteristics of the patient information leaflets (PILs) in French and Spanish; and lastly, we offer some didactic suggestions for the training of medical translators.
\end{abstract}

\section{$1 \quad$ La traducción biosanitaria}

La asignatura de traducción biosanitaria ocupa un lugar destacado en los estudios de Traducción e Interpretación desde su concepción. Sus orígenes se remontan al establecimiento oficial de dichos estudios. Francia es uno de los pioneros en la oferta de dichos estudios (incluida la traducción biosanitaria), en España, sin embargo, es en el año 1991, tal y como lo recoge el Boletín Oficial del Estado (BOE 234 de 30/09/1991)1, cuando se establecen dichos estudios oficialmente como licenciatura y se acuña la asignatura bajo la denominación Traducción especializada.

Las razones por las que esta asignatura se oferta desde el origen de los estudios de Traducción e Interpretación son muy numerosas, aunque la más evidente tiene relación con su componente humano, es decir, con la importancia de las ciencias de la vida y de la salud, de las que participa la sociedad en su conjunto a diversos niveles: médicos, pacientes, investigadores, profesores, alumnos, farmacéuticos, entre otros. Los traductores e intérpretes, miembros de dicho colectivo, son los encargados de velar por la comunicación interlingüística e intercultural en el ámbito biosanitario. Preparar a estos profesionales no es tarea fácil ya que el docente debe tener en cuenta factores como la multidisciplinariedad del campo biosanitario y de la actividad de traducción e interpretación y por supuesto, las características y restricciones de la propia asignatura establecidas por el sistema educativo. Ante este panorama, el docente debe realizar una verdadera obra de ingeniería en la planificación de los contenidos y de las actividades con el fin de que el alumnado adquiera la competencia traductora (cf. Hurtado Albir 2011: 30) en el ámbito biosanitario, que, debiera comprender, en teoría, las siguientes disciplinas: medicina general y sus especialidades, odontología, podología, fisioterapia, biología, farmacología, química, en definitiva, todas las ciencias que se ocupan de algún modo de las ciencias de la salud y de la vida.

\footnotetext{
${ }^{1}$ Véase: www.boe.es/boe/dias/1991/09/30/pdfs/A31773-31775.pdf. 


\section{Objetivos y corpus de trabajo}

El corpus de este trabajo lo ocupa el conocido portal francés de salud Doctissimo.fr y su recién estrenada versión en español. Este sitio web pertenece al grupo Lagardère Active (editorial, televisión y radio) y es el primer portal dedicado al bienestar y a la salud dirigido al gran público. Es una estupenda fuente de documentación para traductores e intérpretes, en la que encontrarán textos paralelos, noticias sobre salud continuamente actualizadas y constituye, asimismo, una óptima fuente terminológica, porque la horquilla temática de la que se compone es muy amplia, principalmente, la versión original francesa. A continuación, mostramos el índice de contenidos que contemplan ambos portales, ordenados tal y como aparecen en línea. Sin embargo, hemos enumerado los contenidos de la versión española, teniendo en cuenta su correspondencia con su original francés:

\section{Doctissimo.fr}

(1) Santé

(2) Médicaments

(3) Grossesse/bébé

(4) Psychologie

(5) Famille

(6) Nutrition

(7) Beauté

(8) Mode

(9) Forme

(10) Sexualité

(11) Recettes
Doctissimo.es

(1) Salud

(2) Medicamentos

- Bienestar

(3) Bebé

(6) Nutrición

(11) Recetas

(7) Belleza

(10) Sexualidad

Tabla 1: Tabla comparativa de contenidos y equivalencias del portal francés de salud y de bienestar, Doctissimo, y de su versión en español.

Dentro de este portal, nos hemos concentrado en la sección de "Medicamentos" ("Médicaments") y más concretamente, en "Guía de medicamentos" ("Guide des médicaments"). En la versión francesa, en esta sección dedicada a los medicamentos, nos ofrecen dos listados con: "los más prescritos" ("Les plus prescrits") y "los más vistos" ("Les plus vus"). Su motor de búsqueda nos permite buscar o bien por "medicamentos" ("Médicament"), "principios activos" ("Principe actif"), "laboratorios" ("Laboratoire") y en la versión española, además, es posible buscar por enfermedades. La guía de medicamentos de la versión original francesa está compuesta por más de 8.000 medicamentos, mientras que la versión española, es más específica y estipula que son 10.882 los medicamentos recogidos en su web. En este trabajo, hemos tomado la versión francesa original como base y para ello, hemos elegido que los medicamentos que van a ser analizados en este estudio tengan como "principio activo" ("Principe actif") principal común "paracetamol" ("Paracétamol"). Tras la búsqueda, encontramos que son 182 en Francia y 227 en España, los medicamentos que tienen como principio activo paracetamol. Para acotar nuestro objeto de estudio y con el ánimo de ser más precisos en nuestros resultados, hemos analizado los 15 primeros medicamentos tanto en Francia como en España por orden alfabético: 

españoles

\begin{tabular}{|c|c|c|c|c|}
\hline \multicolumn{2}{|c|}{ Doctissimo.fr } & & \multicolumn{2}{c|}{ Doctissimo.es } \\
\hline Médicaments & $\begin{array}{c}\text { Clases } \\
\text { thérapeutiques }\end{array}$ & Medicamentos & $\begin{array}{c}\text { Clases } \\
\text { terapéuticas }\end{array}$ \\
\hline $\begin{array}{c}\text { Actifed Etats } \\
\text { Grippaux }\end{array}$ & Allergologie & 1 & Acecat & Analgésicos \\
\hline $\begin{array}{c}\text { Actifed Rhume } \\
\text { Oto-rhino- } \\
\text { laryngologie }\end{array}$ & 2 & Acertol & Analgésicos \\
\hline $\begin{array}{c}\text { Actifed Rhume Jour } \\
\text { et Nuit }\end{array}$ & $\begin{array}{c}\text { Oto-rhino- } \\
\text { laryngologie }\end{array}$ & 3 & $\begin{array}{c}\text { Actron } \\
\text { compuesto }\end{array}$ & Analgésicos \\
\hline $\begin{array}{c}\text { Actifedsign } \\
\text { Actron }\end{array}$ & $\begin{array}{c}\text { Allergologie, } \\
\text { Oto-rhino- } \\
\text { laryngologie }\end{array}$ & 4 & Adalgur & Reumatología \\
\hline Afebryl & Antalgiques & 5 & $\begin{array}{c}\text { Alergical } \\
\text { expectorante }\end{array}$ & Neumología \\
\hline Algicalm & Antalgiques & 6 & Algidol & Analgésicos \\
\hline Algisedal & Antalgiques & 7 & Analgilasa & Analgésicos \\
\hline Algoced Gé & Antalgiques & 9 & Analgiplus & Analgésicos \\
\hline Algodol & Antalgiques & 10 & Anticatarral & Neumología \\
\hline Algodol Cafeine & Antalgiques & 11 & Apiredol & Analgésicos \\
\hline Algotropyl & $\begin{array}{c}\text { Allergologie, } \\
\text { Antalgiques }\end{array}$ & 12 & Apiretal & Analgésicos \\
\hline $\begin{array}{c}\text { Bronalgésicos } \\
\text { Paracétamol }\end{array}$ & $\begin{array}{c}\text { Oto-rhino- } \\
\text { laryngologie }\end{array}$ & 13 & Apiretal codeína & Analgésicos \\
\hline Claradol & Antalgiques & 15 & Auxidor & Analgésicos \\
\hline
\end{tabular}

Tabla 2: Medicamentos franceses y españoles con sus clases terapéuticas y paracetamol como principio activo.

\section{Estructura y características de los prospectos farmacéuticos}

Una vez establecido nuestro objeto de estudio, pasamos a su análisis. Con los 30 medicamentos, que han sido objeto de nuestro estudio, hemos podido observar en qué partes se estructura el prospecto farmacéutico tanto en Francia como en España. Como decíamos anteriormente, hemos partido de la versión original francesa para contrastarlo con la versión española.

En definitiva, la estructura de un prospecto farmacéutico está claramente definida y consta de cinco apartados principales (entre paréntesis, el subapartado correspondiente en español, si lo tiene), de los que derivan numerosos subapartados, como podemos constatar:

\section{1) "Ses indications"}

Éste es el apartado con en el que suele comenzar el prospecto farmacéutico en Francia. Se trata de un breve apartado en el que se describe la función principal del tratamiento en cuestión. No en todos los medicamentos que hemos estudiado, hemos encontrado esta información, al igual que en los prospectos españoles.

\section{2) "Pathologies pour lesquels il peut-être prescrit"}

Este segundo apartado dedicado a la(s) enfermedad(es) o la(s) patología(s) por la(s) que ha sido recetado/prescrito. Normalmente, suele estar ocupado por sustantivos, como: Douleur, Etat 
fébrile (Actron) o incluso, proposiciones en las que se indica el nivel de dolor o de gravedad de una afección, pongamos por caso: Affection douloureuse modérée, Affection douloureuse intense (Algoced Gé). Dicho apartado está divido, a su vez, en varias secciones:

a. "Classe thérapeutique" ("Clase terapéutica"): En esta sección se selecciona la clase terapéutica a la que pertenece dicho medicamento. Doctissimo.fr nos ofrece, a este efecto, un listado con las 25 clases terapéuticas más frecuentes, a saber:

\begin{tabular}{|c|c|c|c|c|c|}
\hline & Doctissimo.fr & Doctissimo.es & & Doctissimo.fr & Doctissimo.es \\
\hline (1) & Allergologie & Alergología & (14) & $\begin{array}{l}\text { Infectiologie- } \\
\text { Parasitologie }\end{array}$ & $\begin{array}{l}\text { Virus, bacterias, } \\
\text { parásitos }\end{array}$ \\
\hline (2) & $\begin{array}{l}\text { Anesthésie, } \\
\text { réanimation }\end{array}$ & $\begin{array}{l}\text { Anestesia, } \\
\text { reanimación }\end{array}$ & (15) & $\begin{array}{l}\text { Métabolisme et } \\
\text { nutrition }\end{array}$ & $\begin{array}{l}\text { Metabolismo y } \\
\text { nutrición }\end{array}$ \\
\hline (3) & Antalgiques & Analgésicos & $(16)$ & $\begin{array}{l}\text { Neurologie- } \\
\text { psychiatrie }\end{array}$ & $\begin{array}{l}\text { Neurología- } \\
\text { psiquiatría }\end{array}$ \\
\hline (4) & $\begin{array}{l}\text { Anti- } \\
\text { inflammatoires }\end{array}$ & Antiinflamatorios & (17) & Ophtalmologie & Oftalmología \\
\hline (5) & $\begin{array}{l}\text { Cancérologie } \\
\text { et hématologie }\end{array}$ & $\begin{array}{l}\text { Cancerología y } \\
\text { hematología }\end{array}$ & (18) & $\begin{array}{l}\text { Oto-rhino- } \\
\text { laryngologie }\end{array}$ & $\begin{array}{l}\text { Otorrinolaringo- } \\
\text { logía }\end{array}$ \\
\hline (6) & $\begin{array}{l}\text { Cardiologie et } \\
\text { angéiologie }\end{array}$ & $\begin{array}{l}\text { Cardiología y } \\
\text { angiología }\end{array}$ & (19) & Pneumologie & Neumología \\
\hline (7) & $\begin{array}{l}\text { Contraception } \\
\text { et interruption } \\
\text { de grossesse }\end{array}$ & $\begin{array}{l}\text { Contracepción e } \\
\text { interrupción del } \\
\text { embarazo }\end{array}$ & $(20)$ & $\begin{array}{l}\text { Produits dia- } \\
\text { gnostiques ou } \\
\text { autres produits } \\
\text { thérapeutiques }\end{array}$ & - \\
\hline (8) & Dermatologie & Dermatología & $(21)$ & Rhumatologie & Reumatología \\
\hline (9) & Endocrinologie & Endocrinología & (22) & Stomatologie & Estomatología \\
\hline (10) & $\begin{array}{l}\text { Gastro-Entéro- } \\
\text { Hépatologie }\end{array}$ & $\begin{array}{l}\text { Gastroenterología } \\
\text { y Hepatología }\end{array}$ & (23) & Toxicologie & Toxicología \\
\hline (11) & Gynécologie & Ginecología & $(24)$ & $\begin{array}{l}\text { Urologie } \\
\text { néphrologie }\end{array}$ & $\begin{array}{l}\text { Urología y } \\
\text { nefrología }\end{array}$ \\
\hline (12) & $\begin{array}{l}\text { Hémostase et } \\
\text { sang }\end{array}$ & $\begin{array}{l}\text { Sangre y } \\
\text { derivados }\end{array}$ & $(25)$ & $\begin{array}{l}\text { Souches } \\
\text { Homéopathiques }\end{array}$ & - \\
\hline (13) & Immunologie & Inmunología & & $\begin{array}{l}- \\
-\end{array}$ & $\begin{array}{l}\text { Cosméticos } \\
\text { Alimentos y } \\
\text { derivados }\end{array}$ \\
\hline
\end{tabular}

Tabla 3: Tabla de clases terapéuticas de Doctissimo en francés y en español, enumeradas de acuerdo al orden y a la equivalencia con la versión francesa.

b. "Principes actifs" ("Principios activos"): Paracétamol, Pseudoéphédrine (Broncorinol Rhume), etc. Estos son compartidos en los prospectos de ambas lenguas y está ocupado por los principios activos propios del medicamento en cuestión.

c. "Excipients" ("ATC"): En la versión española del portal Doctissimo, aparecen las siglas ATC, correspondientes a Anatomical Therapeutic Chemical classification, que hacen referencia al sistema de clasificación europeo (cf. Saladrigas 2004) de medicamentos.

d. "Statut": Este subapartado sólo aparece en los prospectos franceses y hace referencia a la condición en la que podemos conseguir un medicamento. En los casos que hemos estudiado, hemos encontrado dos tipos de informaciones:

- $\quad$ "Ce médicament est NON SOUMIS A PRESCRIPTION MEDICALE $\rightarrow$ (Algodol, Actifed États Grippaux, Actron)". En estos casos, el tratamiento no está sometido a prescripción médica. 

españoles

- "Mode de délivrance/conditionnement modifié/retiré de la vente depuis le 30 juillet 2012 (autres formes pour ce médicament) $\rightarrow$ (Actifedsign, desde el 30 de julio de 2012, Afebryl, desde el 20 de enero de 2004, etc.)" En casos en los que hay una condición especial como: cambio en la forma de envío o en las condiciones o bien, se haya retirado del mercado el medicamento y por lo tanto, se sugieren otros, encontraremos esta información.

e. "Prix de vente TTC": En los prospectos franceses encontramos "Prix de vente TTC (toutes taxes comprises)", que designa el precio de venta incluidos todos los impuestos. En los españoles, aparece un subapartado similar denominado "Precio". La información que se ofrece en esta sección es una cantidad, mediante un número cardinal y el símbolo del euro, para indicar el precio del medicamento o del tratamiento. Por ejemplo: $3 €$ (Acecat), 2,65€ (Claradol Codeine), entre otros.

f. "Tx de remboursement SS": En realidad, "Taux de remboursement de la Sécurité Sociale". Ésta hace referencia a la tasa de reembolso de la Seguridad Social francesa. Este apartado sólo aparece, como es obvio, en los prospectos de la versión original francesa. Al hablar de tasas o impuestos, esta sección conlleva como respuesta un porcentaje: $65 \%$ (Claradol), $0 \%$ (Algicalm), entre otros.

g. "Laboratoire" ("Laboratorio"): Este apartado es compartido en ambas versiones del portal Doctissimo. Los laboratorios dependen de cada país, al igual que la denominación del medicamento, si no es un nombre que tenga que ver con su principio activo primario, es decir, un producto genérico. Es por esta razón, que en nuestro estudio, hemos seleccionado la muestra a analizar, atendiendo a un principio activo principal común para ambos países, como es el paracetamol (paracétamol). Sirvan como ejemplo los siguientes laboratorios: Bayer Santé Familiale (Claradol), Tiedra Farmacéutica, S.L. (Acecat), Fher (Auxidor), entre otros.

\section{3) "Présentation"}

a. "Mode d'administration" (No en todos los medicamentos): Esta información es la relativa al modo de empleo de un tratamiento o medicamento. Pese a su importancia, como hemos podido comprobar con nuestro estudio, estas indicaciones no están disponibles en todos los medicamentos (Broncoline Rhume). En los prospectos españoles, no suele aparecer, porque esta información viene recogida en la Posología ("Posologie").

En el modo de empleo o uso de un tratamiento podemos encontrar informaciones relativas a:

- Tipo de vía: "Voie orale (Cefaline Hauth au Paracétamol)"

- Edad: "Réservé à l'adulte et l'enfant à partir de 15 ans (Cefaline Hauth au Paracétamol)"

- Administración: "Boire après dissolution complète dans un grand verre d'eau (Cefaline Hauth au Paracétamol)"

b. "(Sa) Posologie" ('Posología'): Esta sección está muy relacionada con la que acabamos de ver y a veces, incluso la sustituye. Este apartado de los prospectos recogen la información relativa a:

- Edad: "Réservé à l'enfant pesant entre 13 et $16 \mathrm{~kg}$ (soit environ de 2 à 5 ans) (Algotropyl), entre otros."

- Vía: "Voie rectale (Algotropyl), etc." 
- Información extra (administración y dosis): Esta información tiene que ver con el modo de empleo y de nuevo, aparecerán datos relativos a edades, peso, el número de tomas/dosis, el tiempo o intervalo de tiempo entre una y otra toma, la cantidad de las dosis y su duración. Además, en casos en los que haya una dolencia o se sufra de algún problema adicional, también se indicará el tiempo, cantidad de la dosis, para que no haya ninguna incompatibilidad. Asimismo, si este tratamiento provoca una reacción no deseada, también se indica los cambios en la administración de dicho medicamento o bien, el cese de la aplicación. Veamos toda esta información en un ejemplo. Tomemos el caso del medicamento Algotropyl:

"Chez l'enfant, il est impératif de respecter les posologies définies en fonction du poids de l'enfant et donc de choisir une présentation adaptée. Les âges approximatifs en foction du poids sont données à titre d'information.

La dose quotidienne du paracetamol recommandée dépend du poids de l'enfant: elle est d'environ $60 \mathrm{mg} / \mathrm{kg} / \mathrm{jour}$, à repartir en 4 prises, soit environ 15 $\mathrm{mg} / \mathrm{kg}$ toutes les 6 heures.

En raison du risque et toxicité locale; l'administration d'un suppositoire n'est pas conseillée au delà de 4 fois par jour, et le traitement par voie rectale doit être la plus courte possible.

En cas de diarrhée, l'administration de suppositoire n'est pas recommandée.

La posologie est de 1 suppositoire à $200 \mathrm{mg}$ de paracetamol à renouveler en cas de besoin au bout de 6 heures sans dépasser 4 suppositoires par jour.

Il convient de privilégier les prises vespérales en raison de l'effet sédatif prononcé de la prométhazien, surtout en debut de traitement.

\section{Insuffisance rénale:}

En cas d'insuffisance rénale sévère (clairance de la créantinine inférieure à $10 \mathrm{ml} / \mathrm{min})$, l'intervalle entre deux prises será au minimum de 8 heures $(\mathrm{Al}-$ gotropyl, Doctissimo.fr)"

c. "Son aspect et forme": Esta información sólo la encontramos en algunos de los prospectos franceses que hemos analizado, aunque no en todos (Algodol Cafeine). En este caso, en el que hay una ausencia de esa información, se aclara de la siguiente manera: "Absence d'information dans l'AMM". AMM son las siglas correspondientes a "Autorisation de mise sur le marché", es decir, autorización de venta en el mercado de medicamentos.

En otros tratamientos como, Algodol, sí encontramos dicha información, en este caso: "Comprimé rond blanc sécable gravé PARACÉTAMOL 500 sur une face". En esta sección, se describe físicamente cómo es el producto que vamos a consumir; sin embargo, no la hemos encontrado en los prospectos españoles que hemos analizado.

d. "Sa présentation": Esta sección suele aparecer en los prospectos franceses, aunque no se detalle ninguna información (Algodol, Algoced Gé, Algisedal, Algicalm, Actron, Actifedsign, entre otros). Tampoco consta en los prospectos españoles analizados.

e. "Comment ça marche" ("Mecanismo de acción"): Este apartado, lo encontramos en ambas versiones del portal. Primero, analizaremos las informaciones que encontramos en los prospectos franceses analizados. Veamos el siguiente ejemplo (Actifedsign):

Antalgique antipyretique

Antihistaminique inhibiteur des recepteurs $H_{1}$ 


\section{(N: Système nerveux central)}

\section{( $R$ : Système respiratoire)}

Destacamos de este apartado la especificidad de su terminología, a la que deben prestar especial atención los traductores de prospectos farmacéuticos. No sólo se detallan algunas propiedades del producto o los organismos sobre los que actuará, sino que además, se explica detalladamente su funcionamiento, como veremos con el mismo medicamento (Actifedsign):

"Association de paracetamol, d'un antihistaminique, le maléate de chiorphénamine et de vitamine $\mathrm{C}$.

Maléate de chiorphénamine: antihistaminique $\mathrm{H}_{1}$, à structure propylamine, possédant une activité anticholinergique, à l'origine d'effets indésirables.

Les antihistaminiques $\mathrm{H}_{1}$ ont en commun la propriété de s'opposer, par antagonisme compétitif plus ou moins reversible, aux effets de l'histamine notamment sur la peau, les bronches, l'intestin et les vaisseaux.

Le passage de la barrière hémato-encéphalique est à l'origine d'effets sédatifs, de nature histaminergique et adrénolytique, cette dernière propriété pouvant aussi retentir sur un plan hémodynamique (risque d'hypotension orthostatique) (Actifedsign)"

En el caso de los prospectos españoles, destacamos la concisión de este apartado, con respecto a los franceses. Sirva, a tal efecto, el siguiente ejemplo: "Analgésico y antipirético. Inhibe la síntesis de prostaglandinas en el SNC y bloquea la generación del impulso doloroso a nivel periférico. Actúa sobre el centro hipotalámico regulador de la temperatura (Acertol)"

\section{4) "Précautions"}

a. "Conduite à tenir pour les conducteurs de véhicules": Este apartado va dirigido a los conductores de vehículos o que utilicen/conduzcan otro tipo de maquinaria, porque el uso de medicamentos puede provocar somnolencia (aunque no todos, pongamos por caso, Actron, donde no se da información a los conductores, es decir, Sans objet), sobre todo, en el comienzo del tratamiento y que además, puede acentuarse si se ingiere bebidas alcohólicas u otros medicamentos que contengan alcohol. Éste es el caso de medicamentos como: Actifed États grippaux, Actifed Rhume, entre otros. El mensaje que solemos encontrar es:

"L'attention est appelée, notamment chez les conducteurs de véhicules et les utilisateurs de machines, sur les risques de somnolence attachés à l'emploi de ce médicament surtout en debut de traitement.

Ce phénomène est accentué par la prise de boissons alcoolisées ou de médicaments contenant de l'alcool (Actifed Rhume)."

b. "Ses contre-indications" ("Contraindicaciones"): Esta sección es común para los prospectos de ambas lenguas y está dedicado a las contraindicaciones, es decir, los perjuicios de tomar algún medicamento o la aplicación de algún tratamiento. Éstas varían atendiendo a la composición del medicamento y de los problemas/dolencias previas que el usuario tenga. Por lo tanto, podemos encontrar desde informaciones muy escuetas como por ejemplo: "Hipersensibilidad. Insuficiencia hepatocelular grave" (Acertol), "Afecciones hepáticas" (Adalgur) a informaciones muy extensas en las que se detalle por grupos las contraindicaciones, teniendo en cuenta sus componentes, por ejemplo, en los casos de los prospectos franceses que hemos analizado, siguen esta tendencia. En el caso del medicamento francés Actron, se indican contraindicaciones ligadas a: aspirina ("Liées à l'aspirine"), paracetamol ("Liées au paracetamol"), cafeína 
("Liées à la caféine") y también se desaconseja el propio medicamento o alguno(s) de sus componentes si además se están tratando otras enfermedades o dolencias con otros medicamentos.

Estas informaciones son muy sensibles y de vital importancia para el consumidor del tratamiento. Un error de traducción, que cree una mala interpretación del texto original, puede repercutir directamente en la salud del receptor de nuestro trabajo y por ende, del consumidor del medicamento.

c. "Ses contre-indications" ("Contraindicaciones"): Esta sección es común para los prospectos de ambas lenguas y está dedicado a las contraindicaciones, es decir, los perjuicios de tomar algún medicamento o la aplicación de algún tratamiento. Éstas varían atendiendo a la composición del medicamento y de los problemas/dolencias previas que el usuario tenga. Por lo tanto, podemos encontrar desde informaciones muy escuetas como por ejemplo: "Hipersensibilidad. Insuficiencia hepatocelular grave" (Acertol), "Afecciones hepáticas" (Adalgur) a informaciones muy extensas en las que se detalle por grupos las contraindicaciones, teniendo en cuenta sus componentes, por ejemplo, en los casos de los prospectos franceses que hemos analizado, siguen esta tendencia. En el caso del medicamento francés Actron, se indican contraindicaciones ligadas a: aspirina ("Liées à l'aspirine"), paracetamol ("Liées au paracetamol"), cafeína ("Liées à la caféine") y también se desaconseja el propio medicamento o alguno(s) de sus componentes si además se están tratando otras enfermedades o dolencias con otros medicamentos.

Estas informaciones son muy sensibles y de vital importancia para el consumidor del tratamiento. Un error de traducción, que cree una mala interpretación del texto original, puede repercutir directamente en la salud del receptor de nuestro trabajo y por ende, del consumidor del medicamento.

d. "Ses précautions d'emploi" ("Precauciones"): En esta sección, se ofrecen recomendaciones y avisos con respecto al uso de los medicamentos atendiendo a otras posibles dolencias o enfermedades del consumidor de dicho tratamiento. Veamos algunos ejemplos:

Francés: "En cas de fièvre élevée ou persistante, de survenue de signes de surinfection ou de persistance des symptômes au-delà de 4 jours, une réévaluation du traitement doit être faite (Actifed Rhume Jour et Nuit)"

Español: "Alcohólicos o en caso de I.H.: no sobrepasar 2 g paracetamol/día. Niños $<3$ años. Asmáticos sensibles al AAS (reacción cruzada). Evitar uso prolongado en anemia, afección cardiaca o pulmonar e I.R. grave. Riesgo de daño hepático grave con dosis altas. Vía IV, precaución en malnutrición crónica y deshidratación (Acertol)"

e. "Ses interactions": Esta sección, dedicada a las interacciones, aparece normalmente en los prospectos franceses. En la versión española, esta información suele ir incluida en el epígrafe "6) Effets indésirables" ('Posibles efectos adversos')

En ésta encontramos, posibles problemas derivados del (mal) uso del medicamento. Pongamos por ejemplo: "Interactions avec les examens paracliniques: la prise de paracétamol peut fausser le dosage de l'acide urique sanguin par la méthode à l'acide phosphotungstique, et le dosage de la glycémie par la méthode à la glucose oxydase-péroxydase".

f. "Ses incompatibilités": Éste es otro de los apartados que sí aparecen en la versión original francesa, pero que no está presente en la versión española. En los prospectos 
españoles

españoles, éste se suele fusionar con el apartado "6) Effets indésirables" ('Posibles efectos adversos'), como ocurre con las interacciones.

En algunas ocasiones, en prospectos franceses no se añade información en este apartado (Algisedal), en el que sólo aparece la siguiente frase: "Sans objet".

g. "En cas de surdosage": Esta sección suele aparecer en los prospectos franceses, al contrario que los españoles. En la versión española, esta información suele ir incluida en el apartado 6) Effets indésirables ('Posibles efectos adversos'), tal y como hemos comprobado en los dos apartados anteriores: "d. Ses interactions" y "e. Ses incompatibilités".

Este apartado incluye información sensible respecto a la salud del consumidor de un medicamento, a saber, la información relativa a los casos de sobredosis. Suele ser un apartado extenso (Claradol), porque además puede incluirse: posibles síntomas y conductas de urgencia.

\section{5) "Grossesse et allaitement" ("Embarazo y lactancia")}

Este apartado único en francés, se divide en dos partes: lactancia y embarazo, en español. En ambas versiones, se comparte con el potencial consumidor de dicho medicamento/tratamiento (una mujer embarazada o en periodo de lactancia), los resultados que éste ha tenido en estudios con animales, si estos han sido positivos o si por el contrario, no se recomienda su uso, puesto que de él podrían derivar otros problemas.

\section{6) "Effets indésirables" ("Posibles efectos adversos" o "reacciones adversas")}

Este apartado está ocupado por las enfermedades, reacciones, efectos no deseados producidos por el uso del medicamento, a veces, incluso, se ordenan dichos efectos atendiendo a los componentes del tratamiento. Sirvan para ilustrar sobre esta sección, los siguientes ejemplos:

Francés: "Quelques rares cas de réactions d'hypersensibilité à type de choc anaphylactique, oedème de Quincke, érythème, urticaire, rash cutané ont été rapportés. Leur survenue impose l'arrête définitif de ce médicament et des médicaments apparentés". "De très exceptionnels cas de thrombopénie, leucopénie et neutropénie ont été signalés (Algodol)"

Español: "Raras: malestar, nivel aumentado de transminasas, hipotensión, hepatotoxicidad, erupción cutánea, alteraciones hematológicas, hipoglucemia, piuria estéril (Auxidor)"

\section{Características del lenguaje farmacéutico: prospectos farmacéuticos}

El lenguaje farmacéutico es un lenguaje altamente especializado y multidisciplinar, puesto que está relacionado con otras ciencias como: Medicina, Química, Biología, Farmacología, entre otras. La especificidad de este lenguaje hace que la comunicación entre profesionales de la Farmacología o bien, de la Medicina o de otras disciplinas, sea más precisa, clara, fluida y rápida, de ahí deriva el difícil acceso para el resto de la sociedad. El caso de los prospectos farmacéuticos es muy especial. Este tipo de textos contiene información muy específica, porque en él se describe: un tipo de medicamento y cuáles son sus componentes, ambas secciones contienen una carga terminológica densa, pero además, se ofrecen consejos sobre su modo de empleo y de conservación en los que la lengua general está más presente. Estos son sólo algunos ejemplos que definen claramente a los prospectos farmacéuticos, que son una tipología textual en la que se transmite una información altamente especializada a un usuario que no tiene porqué disponer de conocimientos (avanzados) en ese ámbito. Este usuario común es un consumidor de un producto que le ayudará a mejorar su salud, si lo usa correctamente, tal y como se indica en las indicaciones. Los prospectos farmacéuticos son una de las tipologías textuales que más seguidores tiene. No olvidemos que todos somos potenciales usuarios de tratamientos y de medicamentos, ya que no hay nadie exento de tener algún problema de salud. El traductor tiene 
ante sí una gran responsabilidad al hacer frente esta tipología textual, porque como decíamos anteriormente, un error de traducción puede poner en peligro la salud del receptor de esa traducción y consumidor del medicamento en cuestión.

Anteriormente, hemos visto de qué partes se compone un prospecto francés y español, basándonos en nuestro corpus de trabajo, extraído del portal médico Doctissimo. Ambos recogen el mismo tipo de informaciones, aunque no en el mismo número de apartados o bajo la misma denominación. En definitiva, los prospectos farmacéuticos son las instrucciones del producto (medicamento) que se vende al consumidor, por lo tanto, no pueden faltar informaciones como: presentación del producto, composición, indicaciones, contraindicaciones o incompatibilidades, función, instrucciones de uso y mantenimiento.

Con respecto al lenguaje de los prospectos farmacéuticos destacamos las siguientes características:

- Lenguaje especializado y multidisciplinar:

- Patologías médicas: Rhume, Rhinite, État grippal (Actifed États Grippaux); Artritis reumatoide, Artrosis, Dismenorrea, Dolor, Fiebre, Odontalgias, Dolor musculoesquelético, Cefalea (Acecat).

- Clases terapéuticas: Acide acétylsalicylique, Paracétamol, Acide ascorbique E300 (Afebryl); Analgésicos (Analgiplus).

- Principios activos: Paracétamol, Caféine (Cefaline Hauth au Paracétamol), Clorfenamina maleato, Diprofilina, Guaifenesina, Paracetamol (Alergical expectorante).

- Excipientes: Povidone E1201, Citrique acide E330, Sodium hydrogénocarbonate, Sodium carbonate E500, Mannitol E421, Saccharine sodique E954, Sodium docusate, Sodium benzoate E211, Siméthicone, Arôme cassis: Palmarosa, Propyièneglycol E1520, et. (Claradol), N02BE51+P2 (Analgilasa).

- Números y símbolos: en el caso de los prospectos farmacéuticos que hemos analizado, se incluye el precio $(€)$ y la tasa de reembolso de la Seguridad Social (\%), junto con los prospectos españoles comparte el frecuente uso de cantidades en edades (ans, años), peso $(\mathrm{kg})$, número $(1,2,3,4 \ldots$ prises $)$, frecuencia (par jour, etc.) y cantidad de las dosis (gramos, miligramos o si son comprimidos completos o medios; $1,2,3 \ldots$ comprimés, demi-comprimés, g, mg, entre otros), frecuencia temporal (toutes les 4 heures, etc.), símbolos de mayor $(>)$, menor $(<)$.

- Siglas y abreviaturas: máx. ('máximo'), mín. ('mínimo'), $h$ ('horas'), g ('gramos'), etc.

- Tratamiento de usted: Este recurso es mucho más empleado en español que en francés "Consulte a su médico o farmacéutico si tiene dudas".

- Estilo impersonal: En francés, su uso es muy frecuente: "Il n'est généralement pas nécessaire de dépasser $3 \mathrm{~g}$ de paracétamol par jour soit 6 comprimés à $500 \mathrm{mg}$ par jour", "Les comprimés sont à avaler tels quels avec une boisson (par exemple eau, lait, jus de fruit)". En español, también es un recurso frecuentemente empleado en esta tipología textual: "Es recomendable llevar el envase y el prospecto del medicamento al profesional sanitario".

- Uso de infinitivo con función de imperativo: "Laisser dissoudre complètement le comprimé dans un verre d'eau", "Mantener fuera del alcance y de la vista de los niños". 
- Sustantivaciones: "Traitement symptomatique de l'hypersécrétion nasale au cours des affections aiguës rhinopharyngées avec maux de tête et/ou fièvre", "No recomendado su uso durante el embarazo ya que atraviesa la placenta".

\section{Fuentes documentales para la traducción farmacéutica}

A lo largo de este trabajo, hemos analizado las características textuales y lingüísticas de los prospectos farmacéuticos. El objetivo de este estudio es que sea de utilidad, principalmente, para traductores e intérpretes biosanitarios y otros compañeros, estudiosos de lenguajes de especialidad.

Son muchos los autores, que son cada vez más conscientes, de la importancia del lenguaje farmacéutico, de hecho, a continuación, recomendamos algunas obras que pueden resultar útiles en el estudio o especialización de la traducción de textos farmacéuticos:

- Domínguez-Gil Hurlé/Alcaraz Varó 2005,

- Domínguez-Gil Hurlé/Alcalaz Varó/Martínez 2007,

- García Maldonado 2004,

- Navarro 2002a,

- Navarro 2002b,

- Navarro 2011,

- Saladrigas/Sacristán del Castillo 2004).

\section{Conclusiones}

En este estudio, en el que partimos de la traducción de prospectos farmacéuticos como ámbito profesional de futuro, hemos analizado y contrastado, detalladamente, 30 prospectos farmacéuticos franceses y españoles, extraídos del portal médico francés Doctissimo. De este análisis, hemos obtenido diversas conclusiones. En primer lugar, destacamos su multidisciplinariedad, puesto que los prospectos farmacéuticos comparten términos propios de ramas como la Medicina, la Química, la Biología, entre otras ciencias. Otra característica muy propia de esta tipología textual es su contraste entre lengua general y lenguaje especializado, es decir, la forma en la que se transmite a un usuario de la lengua general, un mensaje cuyo grado de especialización es muy elevado. Asimismo, es digno de mención el grado de responsabilidad que tiene el traductor biosanitario ante este tipo de textos, puesto que una mala interpretación o traducción, supone un potencial riesgo de salud para el usuario de dicha traducción y consumidor del medicamento traducido, al seguir indicaciones erróneas. Esta tipología textual esconde pequeños detalles de los que pueden derivarse traducciones equivocadas, si no se presta la suficiente atención; nos referimos al uso de cifras en las cantidades de las dosis, su frecuencia, edades, entre otros. Las informaciones recogidas en los prospectos farmacéuticos son muy sensibles, puesto que lo que está en juego es la salud del lector de nuestra traducción. Por ello, muchos de los apartados incluidos en los prospectos tienen que ver con instrucciones para los consumidores de los medicamentos, posibles efectos adversos, indicaciones para conductores, mujeres embarazadas o en periodo de lactancia.

Estamos ante una tipología textual que merece la pena seguir investigando, porque es una de las que más lectores puede presumir. En esta línea, nos gustaría subrayar la importancia de dicho sector en ambos países: Francia y España. Son numerosos los laboratorios dedicados al estudio y a la creación de novedosos fármacos, esto sumado al imparable desarrollo tecnológico, hacen de la industria farmacológica una de las que suelen tener más estabilidad y además, mayor proyección laboral. No hay comercialización si no se conocen los nuevos 
productos, es ahí donde la traducción debe actuar. En definitiva, esperamos que este trabajo haya contribuido al estudio del lenguaje farmacéutico y sirva, igualmente, para impulsar nuevas investigaciones en este ámbito.

\section{Bibliografía}

Boletín Oficial del Estado (ed.) (1991): "Real Decreto 1385/1991, de 30 de agosto, por el que se establece el título universitario oficial de Licenciado en Traducción e Interpretación y las directrices generales propias de los planes de estudio conducentes a la obtención de aquél.". BOE 234: 31773-31775. http://www.boe.es/boe/dias/1991/09/30/pdfs/A31773-31775.pdf, consultado 9 de septiembre, 2014.

Doctissimo.es (ed.) (2013): Salud y bienestar - Doctissimo. http://www.doctissimo.eso, consultado 14 de julio, 2013.

Doctissimo.fr (ed.) (2000/2013): Santé et bien être avec Doctissimo. http://www.doctissimo.fr, consultado 14 de julio, 2013.

Domínguez-Gil Hurlé, Alfonso/Alcaraz Varó, Enrique (2005): "Una nueva contribución a la terminología farmacéutica". Tremédica: Panace@. Revista de Medicina, Lenguaje y Traducción 6/20: 191-192. http://www.medtrad.org/panacea/IndiceGeneral/n20_resenas_ dominguez-alcarazvaro.pdf, consultado 19 de agosto, 2014.

Domínguez-Gil Hurlé, Alfonso/Alcaraz Varó, Enrique/Martínez Motos, Raquel (2007): Diccionario terminológico de ciencias farmacéuticas. Inglés-español/Spanish-English. Barcelona: Ariel y Real Academia Nacional de Farmacia.

García Maldonado, José T. (ed.) (2004): Diccionario del medicamento Farma-Term. Madrid: El Alcuzón.

Hurtado Albir, Amparo (2011): Traducción y traductología. Introducción a la Traductología. 5. ed. Madrid: Cátedra.

Navarro, Fernando A. (2002a): "Enmienda al diccionario de fármacos". Tremédica: Panace@. Revista de Medicina, Lenguaje y Traducción 3/8: 6. http://www.medtrad.org/ panacea/IndiceGeneral/n8-NavarroCartas.pdf, consultado 19 de agosto, 2014.

Navarro, Fernando A. (2002b): "Glosario de fármacos con nombre común no internacional (EN-ES)". Tremédica: Panace@. Revista de Medicina, Lenguaje y Traducción. 3/7: 10-24. http://www.medtrad.org/panacea/IndiceGeneral/n7_GlosarioFarmacos.pdf, consultado 19 de agosto, 2014.

Navarro, Fernando A. (2011): "Darreichungsformen-Abkürzungen DE-ES: glosario de claves trilíteras utilizadas para indicar la forma galénica en bases farmacéuticas de datos". Tremédica: Panace@. Revista de Medicina, Lenguaje y Traducción 12/34: 200-217. http://www.medtrad.org/panacea/IndiceGeneral/n34-tradyterm-navarro2.pdf, consultado 19 de agosto, 2014.

Saladrigas, María V. (2004): "Sistema de clasificación ATC de sustancias farmacéuticas para uso humano (The Anatomical, Therapeutic, Chemical Classification System". Tremédica: Panace@. Revista de Medicina, Lenguaje y Traducción 5/15: 58-60. http://www.medtrad.org/panacea/IndiceGeneral/n15_tribuna-Saladrigas.pdf, consultado 19 de agosto, 2014.

Saladrigas, María V./Sacristán del Castillo, José A. (2004): "Fichas de MedTrad (n. ${ }^{\circ}$ 13): eficacia, efectividad y eficiencia en la investigación de fármacos". Tremédica: Panace@. Revista de Medicina, Lenguaje y Traducción. 5/17-18: 188-190. http://www.medtrad.org/ panacea/IndiceGeneral/n17-18 tradyterm-Saladrigas.pdf, consultado 19 de agosto, 2014. 